\title{
Endosomal escape enhancing compounds facilitate functional delivery of extracellular vesicle cargo
}

\author{
Nikki Heath ${ }^{1}$ (D), Xabier Osteikoetxea ${ }^{1}$, Taiana Mia de Oliveria², Elisa Lázaro-lbáñez ${ }^{3}$, Olga \\ Shatnyeva ${ }^{3}$, Christina Schindler ${ }^{4}$, Natalie Tigue ${ }^{4}$, Lorenz M Mayr ${ }^{5}$, Niek Dekker ${ }^{3}$, Ross \\ Overman $^{1}$ \& Rick Davies*,1 \\ ${ }^{1}$ Discovery Biology, Discovery Sciences, BioPharmaceuticals, R\&D, AstraZeneca, Alderley Park, UK \\ ${ }^{2}$ Structure, Biophysics \& FBLG, Discovery Sciences, Biopharmaceuticals R\&D, Cambridge, UK \\ ${ }^{3}$ Discovery Biology, Discovery Sciences, BioPharmaceuticals R\&D, AstraZeneca, Gothenburg, Sweden \\ ${ }^{4}$ Antibody Discovery \& Protein Engineering, BioPharmaceuticals R\&D, AstraZeneca, Granta Park, Cambridge, UK \\ ${ }^{5}$ GE Healthcare Life Sciences, The Grove Centre, White Lion Road, Amersham, UK \\ *Author for correspondence: Rick.Davies@astrazeneca.com
}

Aim: Extracellular vesicles (EVs) are desirable delivery vehicles for therapeutic cargoes. We aimed to load EVs with Cre recombinase protein and determine whether functional delivery to cells could be improved by using endosomal escape enhancing compounds. Materials \& methods: Overexpressed CreFRB protein was actively loaded into EVs by rapalog-induced dimerization to CD81FKBP, or passively loaded by overexpression in the absence of rapalog. Functional delivery of CreFRB was analysed using a HEK293 Cre reporter cell line in the absence and presence of endosomal escape enhancing compounds. Results: The EVs loaded with CreFRB by both active and passive mechanisms were able to deliver functional CreFRB to recipient cells only in the presence of endosomal escape enhancing compounds chloroquine and UNC10217938A. Conclusion: The use of endosomal escape enhancing compounds in conjunction with EVs loaded with therapeutic cargoes may improve efficacy of future EV based therapeutics.

First draft submitted: 8 February 2019; Accepted for publication: 22 August 2019; Published online: 14 November 2019

Keywords: chloroquine $\bullet$ Cre recombinase $\bullet$ endosomal escape $\bullet$ exosomes $\bullet$ extracellular vesicles $\bullet$ rapalog-induced dimerization • therapeutic delivery • UNC10217938A

Efficient delivery of therapeutic agents to cells for the treatment of disease can be challenging. Nanoparticle liposome formulations are popular therapeutic delivery vehicles. They can be modified to increase uptake of therapeutic agents into cells, facilitate their endosomal escape and reduce their uptake by phagocytic cells to increase the efficiency of cargo delivery [1,2]. While extensive research has been carried out to package and deliver nucleic acids such as siRNA using liposome formulations [3], less research has been performed on liposomal delivery of protein cargoes [4]. Important limitations of liposome formulations include toxicity, immunostimulation, complement activation, limited ability to penetrate tissues outside the reticuloendothelial system and lack of batch-to-batch reproducibility [2,5-7]. Therefore, research efforts are now focused on new therapeutic possibilities to overcome the drawbacks of current liposomal formulations.

Extracellular vesicles (EVs) are promising nanoparticle therapeutic delivery candidates. EVs are lipid bilayer enclosed vesicles released by most cell types either by budding directly from the plasma membrane (microvesicles) or by fusion of multivesicular bodies with the plasma membrane to release exosomes into the extracellular environment $[8,10]$. EVs contain lipids, proteins and nucleic acids (e.g., DNA, mRNA and miRNA) from the cell of origin [9-11], and are well documented to efficiently deliver functional protein and nucleic acid cargoes to cells. Therefore, EVs have an important role in autocrine and paracrine intercellular communication to modify cellular phenotypes in health and disease [9-17].

The innate ability of EVs to transfer functional cargoes between cells make them ideal therapeutic cargo carriers. Furthermore, recent studies have shown that adherent and suspension HEK293 (Human Embryonic 
Kidney cells) derived cell lines release EVs that have low immunogenicity and toxicity in vitro and in vivo $[18,19]$. Additionally, the presence of CD47 on siRNA loaded EVs has been reported to decrease macrophage mediated immune clearance of EVs giving them a longer half-life to deliver their therapeutic cargo [20]. These studies indicate that cell line derived non-toxic and non-immunogenic EVs could be used in place of immunogenic liposomes for delivery of therapeutic agents.

Research into the use of EVs as delivery vehicles of therapeutic small molecules, proteins, DNA, mRNA, miRNA and siRNA is rapidly growing. EVs have been used to deliver chemotherapeutic agents such as doxorubicin and paclitaxel to cancer cells [21-24]. Plasmid DNA has been loaded into EVs by forming hybrid vesicles between liposomes and exosomes [25], siRNA has been loaded into EVs by electroporation [20,26] and catalase mRNA has been packaged into EVs using EXOtic exosomes [27].

There is a large repertoire of work describing the packaging and delivery of nucleic acids using EVs. Fewer studies have assessed the use of EVs as nanocarriers of therapeutic proteins. Exogenous loading of proteins into EVs after isolation is challenging. Consequently, engineered cell lines have been designed to actively load proteins into EVs. One such system utilized the late domain pathway, in which WW tagged Cre recombinase resulted in ubiquitination and loading of Cre into EVs [28]. In a separate system, Cre recombinase has been inducibly loaded into EVs using light-induced dimerization [29]. Finally, FKBP rapamycin/rapalog induced dimerization to FRB domain of mTOR was used to load Cas9 into virus-like particles (VLP) termed 'gesicles' [30-33].

For efficient delivery of therapeutic cargoes by EVs, uptake into recipient cells is necessary. The uptake of EVs can occur in several ways including membrane fusion [34], macropinocytosis [35,36] and endocytosis [36,37] - among others [37,39]. There is evidence that EVs taken up by endocytosis can be found within endosomes and lysosomes [40]. If EVs are unable to escape the endosomal compartment, they could instead be degraded by lysosomes impairing functional delivery of EVs and their cargoes.

There are several reagents or compounds that can be used to enhance the uptake and endosomal escape of cargoes by different mechanisms. For example, amphotericinB increases cell membrane permeability [41]. Chloroquine [4244], amantadine [44] and ammonium chloride [44] are lysosomotropic endosomal escape enhancing compounds. Chloroquine enhances endosomal escape by the proton sponge effect. Membrane permeable chloroquine is protonated during endosome maturation resulting in $\mathrm{Cl}^{-}$and $\mathrm{H}_{2} \mathrm{O}$ influx, endosome swelling and rupture releasing endosomal cargoes into the cytoplasm [44]. On the other hand, bafilomycinA inhibits endosome acidification and maturation by inhibiting proton transport into the endosome. This results in accumulation of endosomal cargo and inhibition of cargo escape and delivery [45]. The compound UNC10217938A increases endosomal escape and functional delivery of antisense oligonucleotides (ASOs) [46], and the solution iTOP enhances the uptake of proteins by macropinocytosis and aids the escape of proteins from macropinosomes [47]. Finally, EGF increases EV uptake by macropinocytosis in oncogenic K-RAS expressing cells [35].

Here we evaluate active loading of $E V s$ with CreFRB protein using rapalog controlled protein heterodimerization of overexpressed CreFRB and CD81FKBP, as compared with passive loading of EVs in the absence of rapalog dimerizing ligand. We then assess the efficiency of $\mathrm{EV}$ mediated functional delivery of CreFRB to recipient cells, and investigate the role of endosomal escape enhancing compounds in improving functional delivery of $\mathrm{EV}$ cargoes.

\section{Materials \& methods}

Cells \& cell culture

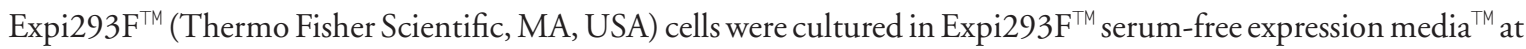
$37^{\circ} \mathrm{C}$ in $8 \% \mathrm{CO}_{2}$ using erlenmeyer flasks at $125 \mathrm{rpm}$ with a $230 \mathrm{~mm}$ throw. HEK293-loxP-GFP-RFP (puromycin) Cre reporter cells (Amsbio, Abingdon, UK) were cultured in high glucose DMEM, $2 \mathrm{mM}$ glutamine, 10\% FCS (all Thermo Fisher Scientific) and $5 \mu \mathrm{g} / \mathrm{ml}$ Puromycin (Sigma-Aldrich, MO, USA) at $37^{\circ} \mathrm{C}$ in $5 \% \mathrm{CO}_{2}$. These will be referred to as HEK293 Cre reporter cells from here on in.

\section{Transfection of EV producing cells}

For transfection of EV producing cells with CD81FKBP and CreFRB, $40 \mathrm{kDa}$ PEI max (polysciences) transfection reagent was prepared at $1 \mathrm{mg} / \mathrm{ml}$, sterile filtered $(0.2 \mu \mathrm{m})$ and stored at $-20^{\circ} \mathrm{C}$. Expi293 $\mathrm{F}^{\mathrm{TM}}$ cells were seeded at $2 \times 10^{6}$ cells $/ \mathrm{ml}, 24$ hours later PEI max-DNA complexes were incubated at room temperature for $15 \mathrm{~min}$ and added to cells. The ratios used were $25 \mathrm{ml}$ cells, $100 \mu \mathrm{l}$ PEI max and $25 \mu \mathrm{g}$ total DNA; this could be scaled up/down accordingly. Approximately $24 \mathrm{~h}$ after transfection, cells were pelleted by centrifugation at $300 \times \mathrm{g}$ for 10 min to remove excess PEI-max-DNA complexes. The cell pellet was washed with phosphate-buffered saline (PBS), 
the centrifugation step repeated and the cell pellet resuspended in double the transfection volume of Expi293 $\mathrm{F}^{\mathrm{TM}}$ expression media. Cells conditioned the media for $48 \mathrm{~h}$ before EVs were harvested. For rapalog-induced loading of EVs, CreFRB and CD81FKBP transfected cells were incubated with (active loading) or without (passive loading) rapalog AP21967 (Takara A/C heterodimerizer) during the $48 \mathrm{~h} \mathrm{EV} \mathrm{media} \mathrm{conditioning} \mathrm{period.} \mathrm{Further} \mathrm{expression}$ plasmid information is available in Supplementary Figure 1.

\section{EV collection}

Conditioned media was centrifuged at $300 \times \mathrm{g}$ for $10 \mathrm{~min}$ to pellet cells and then at $2500 \times \mathrm{g}$ for $10 \mathrm{~min}$ to pellet dead cells and debris. Remaining supernatant was then distributed into $38 \mathrm{ml}$ polyallomer centrifuge tubes (Beckman Coulter) and centrifuged using a SW32 Ti rotor, at 10,000 $\times \mathrm{g}$ for $30 \mathrm{~min}$ to pellet apoptotic bodies and larger microvesicles. The final EV pellet was isolated by a $100,000 \times \mathrm{g}$ spin for $70 \mathrm{~min}$. Supernatant was discarded and pellets (pooled if from multiple tubes) were washed in $1.5 \mathrm{ml}$ PBS (Sigma-Aldrich). The resultant EV solution was transferred to a $1.5 \mathrm{ml}$ Eppendorf tube. This was then floated in the larger polyallomer tube containing $35 \mathrm{ml}$ PBS for a final spin at $100,000 \times \mathrm{g}$. The resultant pellet was resuspended in a final volume of $100-300 \mu \mathrm{l}$ of PBS for further analysis.

\section{Western blotting}

Up to $6 \times 10^{6}$ cells were pelleted by centrifugation at $300 \times \mathrm{g}$, the cell pellet was washed with PBS and repelleted using a final $300 \times$ g centrifugation for $5 \mathrm{~min}$. The cell pellet was then lysed in $50 \mathrm{mM}$ Tris/1\% SDS lysis buffer, homogenized using a $23 \mathrm{G}$ needle and centrifuged at $13,000 \times \mathrm{g}$ at $4^{\circ} \mathrm{C}$. Supernatant was retained for western blotting. Lysate/EV protein concentration was determined by NanoDrop ${ }^{\mathrm{TM}}$ spectrophotometer (Thermo Fisher Scientific) and BCA assay carried out according to manufacturer's instructions (Thermo Fisher Scientific). Lysate/EV samples were mixed with four-times LDS sample buffer (NuPAGE Thermo Fisher Scientific) plus 10\% $\beta$-mercaptoethanol (Sigma-Aldrich) and boiled for $10 \mathrm{~min}$.

Protein samples were ran on $4-12 \%$ gradient Bis/Tris 15 well gels (NuPAGE Thermo Fisher Scientific) in Mops buffer ( $50 \mathrm{mM}$ Tris, $50 \mathrm{mM}$ MOPS, 0.1\% SDS, $1 \mathrm{mM} \mathrm{EDTA)}$ at $170 \mathrm{mV}$ for $1 \mathrm{~h}$. SeeBlue ${ }^{\mathrm{TM}}$ Plus2 and MagicMark $^{\top \mathrm{TM}}$ (Thermo Fisher Scientific) protein standards were used. Proteins were transferred onto nitrocellulose membrane using iBlot ${ }^{\circledR}$ system according to manufacturer instructions (Thermo Fisher Scientific). Subsequently, the membrane was blocked with milk or BSA in TBS-T ( $100 \mathrm{mM}$ Tris- $\mathrm{HCl}, 150 \mathrm{mM} \mathrm{NaCl}$ and $0.5 \%$ Tween) for $1 \mathrm{~h}$ at room temperature. Membranes were incubated with primary antibodies overnight with agitation at $4{ }^{\circ} \mathrm{C}$ in $1 \%$ blocking buffer in TBST-T. After three washes with TBS-T, the secondary HRP conjugated antibodies were applied to membranes for $1 \mathrm{~h}$ at room temperature with agitation. After three washes, the membrane was developed using ECL extreme developing reagent (Expedion) according to manufacturer's instructions. Images were acquired using Bio-Rad ChemiDoc ${ }^{\top M}$ imaging system and the Image Lab 5.0 software. For specific primary antibody and blocking reagent information please see Supplementary Table 1.

\section{Nanoparticle tracking analysis}

LM10 NanoSight (Malvern Instruments, Malvern, UK) with a $405 \mathrm{~nm}$ laser was used according to manufacturer instructions to measure the size and concentration of EVs. For each sample, three $30 \mathrm{~s}$ measurements were acquired using camera level 14 and detection threshold 4 of each sample. Data collection and analysis software version were NTA 3.2 Dev Build 3.2.16.

\section{Compound screen}

For the compound screen, 5000 HEK293-loxP-GFP-RFP (puromycin) Cre reporter cells (Amsbio) per well were plated in a 384-well plate in a total volume of $50 \mu \mathrm{l} /$ well. After $24 \mathrm{~h}$, cell culture supernatant was removed and replaced with $5.2 \mu \mathrm{g} /$ well TATCre and the following treatment conditions in triplicate wells: chloroquine 200-25 $\mathrm{MM}$ (Sigma-Aldrich), ammonium chloride 10-1 mM (Sigma-Aldrich), bafilomycinA 200-50 nM (SigmaAldrich), EGF $1 \mu \mathrm{M}-100 \mathrm{nM}$ (Sigma-Aldrich), amantadine 1.5-0.5 mM (Sigma-Aldrich), amphotericinB 50$10 \mu \mathrm{M}$ (Sigma-Aldrich), UNC10217938A 10-1 $\mu \mathrm{M}$ (WuXi Pharma Tech Inc., Shanghai, China), EGF $1 \mu \mathrm{M}-$ $100 \mathrm{nM}$ and iTOP five-times buffer as described by D'Astoflo et al. (2015); $500 \mathrm{mM} \mathrm{NaCl}, 25 \mathrm{mM} \mathrm{NaH} \mathrm{PO}_{4}$, $250 \mathrm{mM}$ NDSB-201, $150 \mathrm{mM}$ glycerol, $75 \mathrm{mM}$ glycine, $1.25 \mathrm{mM} \mathrm{MgCl}_{2}, 1 \mathrm{mM}$ 2-mercaptoethanol (all chemicals from Sigma-Aldrich), $\mathrm{pH}$ 8, filter sterilized with $0.2 \mu \mathrm{m}$ filter (Thermo Fisher Scientific) [47]. Plates were imaged on the IncuCyte ${ }^{\circledR} S 3$ every $2 \mathrm{~h}$ and the 24 -h time point was chosen for analysis. Image analysis was done using 
the IncuCyte ${ }^{\circledR}$ S3 software S3 2018. Due to the long half-life of GFP protein, at $24 \mathrm{~h}$ GFP was still expressed by most of the cells. As determined by IncuCyte ${ }^{\circledR}$ S3 software, cells were approximately $20-40 \%$ confluent at point of analysis. A mask was created for both GFP and RFP, the data were expressed as the percentage of RFP positive cells (positive indication for Cre recombination) from the total population of GFP expressing cells. As the mask for the bright RFP fluorescence could sometimes exceed $100 \%$ - data were normalized to a maximum of $100 \%$ using GraphPad Prism 8.0.1 (Figure 1A).

Treatment of cells with EVs \& endosomal escape enhancing compounds

Approximately 5000 Cre reporter cells were plated into 384-well plates and approximately $24 \mathrm{~h}$ later, media was removed and replaced with $50 \mu \mathrm{l}$ media containing $20 \mu \mathrm{g} / \mathrm{ml} \mathrm{EVs}(1 \mu \mathrm{g} \mathrm{EV} /$ well/10,000 cells - assuming a 24-h cell doubling time), and the desired concentration of compound. Each condition was done in triplicate wells. Cells were imaged using IncuCyte ${ }^{\circledR}$ S3 for 24 h every 2 h. The 24-h time point was used for image analysis using IncuCyte ${ }^{\circledR}$ S3 software S3 2018 as described in the compound screen method.

\section{Trypsin \& Triton X-100 treatment of EVs}

For western blot experiments, $10 \mu \mathrm{g}$ of CreFRB loaded EVs were treated with or without $0.1 \%$ Triton X-100 (Sigma-Aldrich) for $30 \mathrm{~min}$ at room temperature to induce vesicle membrane lysis as described previously [48]. The EVs were then treated with or without $0.008 \% / 3 \mu \mathrm{M}$ trypsin (2.5\% trypsin; Thermo Fisher Scientific) for 40 min at room temperature. Subsequently, $4 \times$ LDL loading buffer was added to the EVs and they were boiled at $95^{\circ} \mathrm{C}$ for $20 \mathrm{~min}$ to deactivate any further trypsin activity before $2.5 \mu \mathrm{g} /$ well was used for western blots. Three biological replicate EV samples were analyzed together on the same western blot. To control that Cre is delivered by membrane enclosed EVs and not soluble contaminants, concentrated CreFRB loaded EVs and TATCre were treated with or without $0.1 \%$ Triton X-100 for $30 \mathrm{~min}$. Next, CreFRB EVs and TATCre from both treatment conditions were added to HEK293 Cre reporter cells resulting in final concentrations of $1 \mu \mathrm{g} / \mathrm{well}(\mathrm{at} 20 \mu \mathrm{g} / \mathrm{ml})$ for EVs and $325 \mathrm{ng} /$ well for TATCre. Due to the high dilution of the treated EV stock and TATCre samples, the final concentrations of Triton X-100 in cell media were below $0.0025 \%$ which showed no evidence of cell death or morphological changes compared with Triton X-100 free conditions.

\section{Cryo-electron microscopy}

A drop of $2.5 \mu \mathrm{l}$ of each EV sample was applied to a freshly glow discharged 1.2/1.3 Quantifoil Holey Grid - 300 mesh coated with a $2 \mathrm{~nm}$ thick carbon film. Glow discharging was performed for $1 \mathrm{~min}$ in 0.2 bar air pressure. The vitrification procedure was conducted with the aid of Vitrobot Mark IV (FEI Company, OR, USA) at $4{ }^{\circ} \mathrm{C}$ and $100 \%$ humidity. Blotting time was set to $1 \mathrm{~s}$ after which the grids were plunged into liquid ethane. Imaging of the grids was performed on a Titan Krios electron microscope (FEI Company) operated at $300 \mathrm{kV}$. Images were recorded using the integration mode of a Falcon III camera (FEI Company) with a pixel size of $0.14 \mathrm{~nm}$ at $5 \mu \mathrm{m}$ defocus.

\section{Nuclease digestion of EVs}

A total of $20 \mu \mathrm{g}$ EVs were digested with nuclease Basemuncher (Expedion) which was used at 1:10,000 $(2.5 \mathrm{U} / 10 \mathrm{ml})$ in PBS with $2 \mathrm{mM} \mathrm{MgCl} 2$ in a $30 \mu \mathrm{l}$ reaction volume. Reactions were incubated for $30 \mathrm{~min}$ at $37^{\circ} \mathrm{C}$. Digested EVs were resuspended in $1.5 \mathrm{ml} \mathrm{PBS}$ and centrifuged at $100,000 \times \mathrm{g}$ for $70 \mathrm{~min}$. Supernatant was removed and the pellet resuspended in $20 \mu \mathrm{lPBS}(1 \mu \mathrm{g} / \mu \mathrm{l} \mathrm{EV})$. The EVs were then used in further experiments.

\section{Data analysis}

Figures were made using Inkscape 0.92. Data were handled in Excel and analyzed in GraphPad Prism7 and 8.0.1. Data were tested for normal distribution and if normal, parametric analysis was carried out using student's t-test and one-way analysis of variance (ANOVA). Where data distribution was not normal, Mann-Whitney and KruskalWallis tests were undertaken instead. Asterisks in figures represent level of significance: ${ }^{*} \mathrm{p} \leq 0.05,{ }^{* *} \mathrm{p} \leq 0.01$, ${ }^{* * *} \mathrm{p} \leq 0.001$ and $^{* * * *} \mathrm{p} \leq 0.0001$. 


\section{Results}

Endosomal escape enhancing compounds increase the delivery of TATCre to recipient cells

We aimed to explore the usefulness of uptake enhancing or endosomal escape enhancing agents to facilitate functional delivery of EV therapeutic cargoes. To identify the most promising compounds to test, we employed a cell-based reporter system to measure the functional delivery of Cre recombinase; HEK293 cells expressing GFP that respond to Cre recombinase by expressing RFP. In order to prioritize compounds that may benefit EV cargo delivery, a more accessible reagent TATCre (cell penetrating peptide [TAT] containing Cre recombinant protein) delivery was assessed in the presence of compounds that could enhance uptake and/or endosome/macropinosome escape.

Approximately 10,000 HEK293 Cre reporter cells were treated with $5.2 \mu \mathrm{g}$ TATCre in the absence and presence of chloroquine, amphotericinB, amantadine, ammonium chloride, UNC10217938A, EGF, iTOP, negative control bafilomycinA and compound solution controls. After $24 \mathrm{~h}$ of compound treatment, the percentage confluence of GFP cells was analyzed using IncuCyte ${ }^{\circledR}$ S3 software S3 2018 software. Some compound treatments decreased or increased cell confluence suggesting that compounds may effect cell proliferation and/or viability (Supplementary Figure 2). The efficiency of TATCre recombinase activity was analyzed by calculating the percentage of GFP positive cells that were also expressing RFP (positive indication of Cre recombinase activity). We found that $50 \mu \mathrm{M}$ chloroquine and $5 \mu \mathrm{M}$ of the UNC10217938A compound were effective at increasing the functional delivery of TATCre to the HEK293 Cre reporter cell line (Figure 1A \& B). Additionally, $10 \mu \mathrm{M}$ of UNC10217938A also enhanced functional delivery of TATCre; however, it had a negative impact upon cell morphology after $24 \mathrm{~h}$ of treatment. Other compounds that demonstrated a statistically significant effect included amphotericin B, amantadine and iTOP. As expected the control DMSO, water and acetic acid conditions did not enhance TATCre functional delivery above the baseline observed upon TATCre delivery alone (red dotted line), demonstrating that any significant effects observed were attributed to the compounds.

The most effective compounds chloroquine $(50 \mu \mathrm{M})$ and UNC10217938A $(5 \mu \mathrm{M})$ were found to increase Cre-recombinase activity up to $95 \%$ even when TATCre was titrated from $5.2 \mu \mathrm{g}$ to $325 \mathrm{ng} / \mathrm{well} / 10,000 \mathrm{cells}$ (Supplementary Figure 3A). A total of $325 \mathrm{ng} /$ well/10,000 cells TATCre was used in subsequent experiments. However, $50 \mu \mathrm{M}$ chloroquine was toxic to the cells with rounded morphology visualized and a decreased percentage confluence measured at $24 \mathrm{~h}$ (Supplementary Figure 3B). We titrated the chloroquine concentration to determine the lowest concentration of chloroquine which could achieve high efficiency of Cre recombination with a low effect on cell toxicity. This was found to be $25 \mu \mathrm{M}$ at which cell morphology appeared to remain spread at $24 \mathrm{~h}$, although cell confluence was still decreased (Supplementary Figure 4).

\section{Chloroquine \& UNC10217938A increase the functional delivery of CreFRB by EVs}

To assess the usefulness of chloroquine and UNC10217938A in the functional delivery of EV cargoes, we actively loaded EVs with Cre recombinase by rapalog-induced dimerization. Two chimeric proteins, CD81FKBP and $\mathrm{CreFRB}^{\mathrm{T} 2098}$, were cotransfected and overexpressed in suspension Expi293 $\mathrm{F}^{\mathrm{TM}}$ cells. Upon addition of the rapalog AP21967, the FKBP and FRB protein domains dimerize, bringing the CreFRB cargo into the vicinity of membrane protein CD81 where EV biogenesis is likely to take place (Figure 2A). Western blots showed that the presence of heterodimerizing rapalog AP21967 was essential for efficient active loading of CreFRB into EVs (Figure 2B \& Supplementary Figure 5). Two main bands were observed, the band with a higher molecular weight is CreFRB $(\sim 40 \mathrm{kDa})$ and the lower molecular weight band (10 kDa smaller) may be a cleavage by-product of Cre without the FRB domain. CreFRB was minimally loaded into EVs in the presence of ethanol vehicle control with only faint bands observed by passive loading (an overexposed blot exemplifying passively loaded CreFRB can be found in Supplementary Figure 10). From here on in the EVs will be referred to as passively loaded (in the presence of vehicle) and actively loaded (in the presence of rapalog dimerizing compound). Overexpression of CD81FKBP and CreFRB decreased cell proliferation (Supplementary Figure 6A) increased the number of EVs released per cell (Figure 2C) (although not significantly), and did not alter the average size of EVs released (Figure 2D \& Supplementary Figure 6B). The observed increase in $\mathrm{EV}$ release is not altogether surprising, as previous studies have described a role of tetraspanins in EV release (CD9 and CD82) [49] and EV loading (CD81) [50].

A ligand titration of rapalog AP21967 was carried out to determine efficiency of Cre loading at lower doses to avoid any off-target effects of the rapalog. Off target effects of rapalog compounds at high concentrations could interfere with the mTOR pathway, which is involved in multiple cellular processes such as autophagy, protein synthesis, cell survival and proliferation [51]; therefore, the use of excess rapalog is a valid concern. Supplementary 


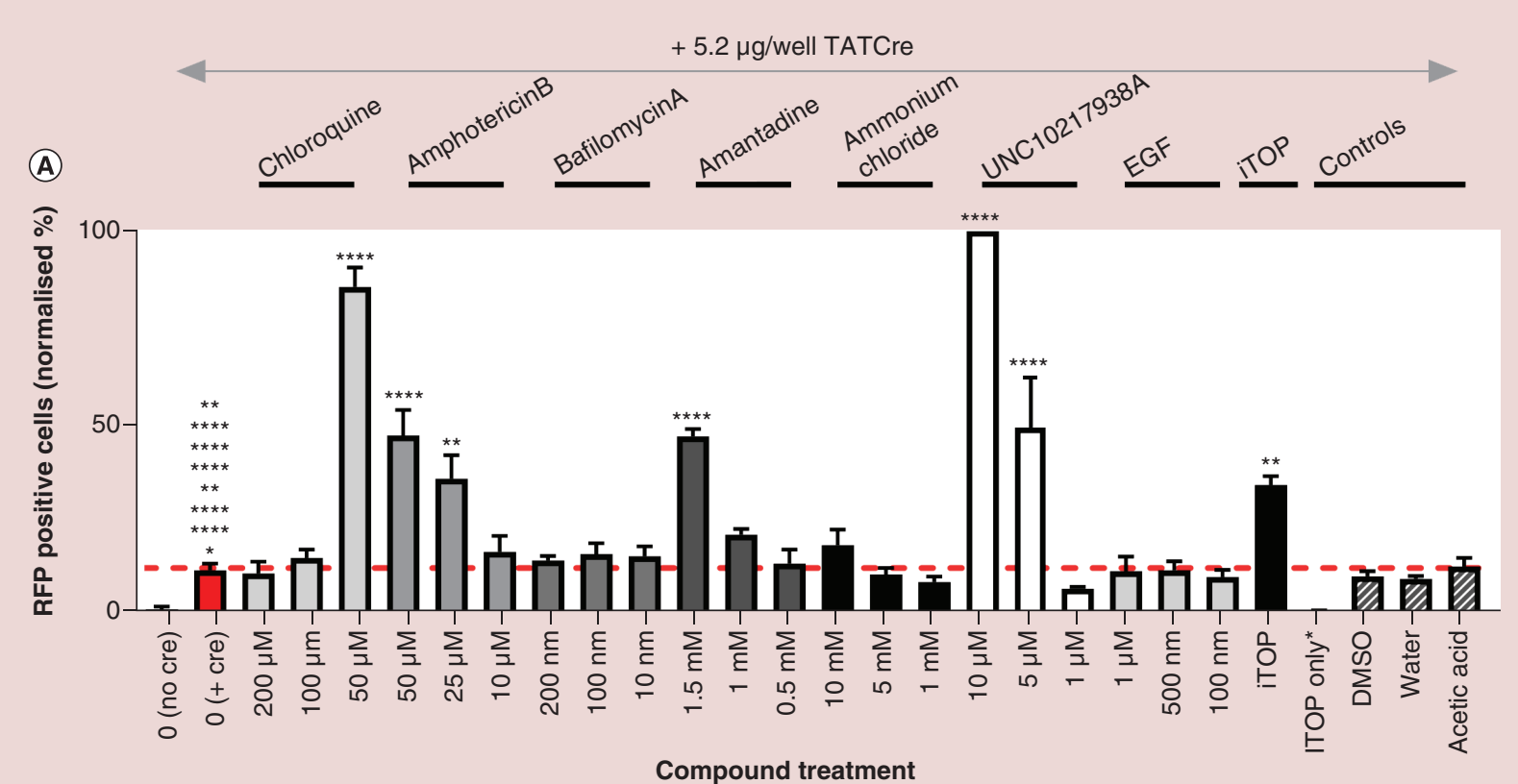

(B)
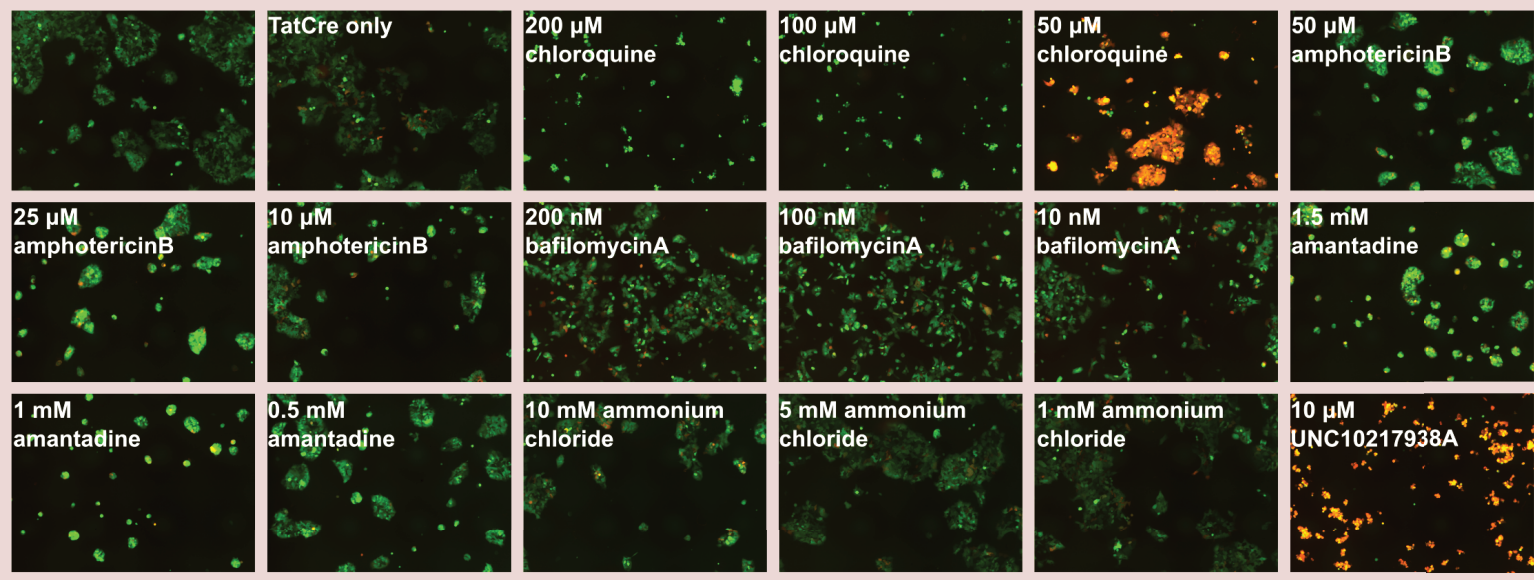

bafilomycinA
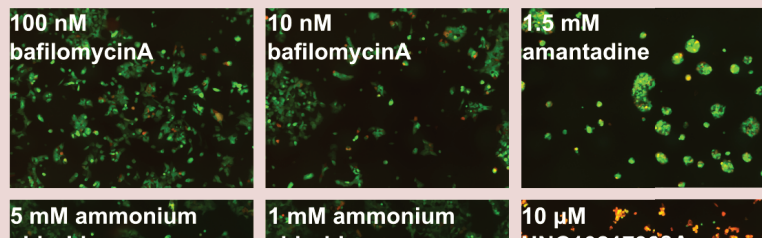

chloride
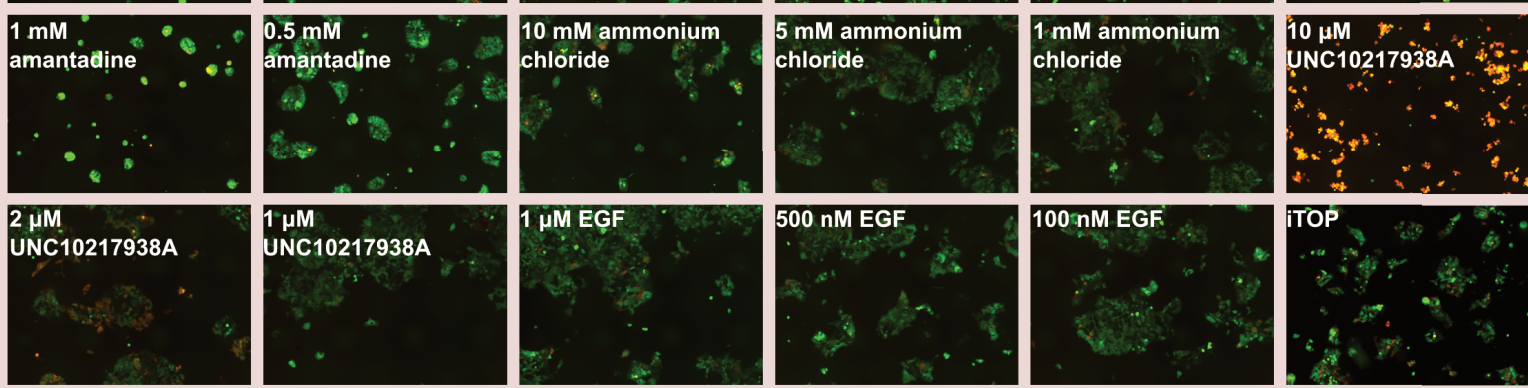

$1 \mu \mathrm{M}$

UNC10217938A
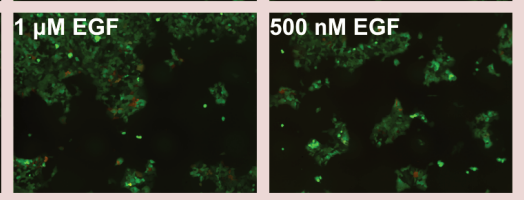

100:nM EGF
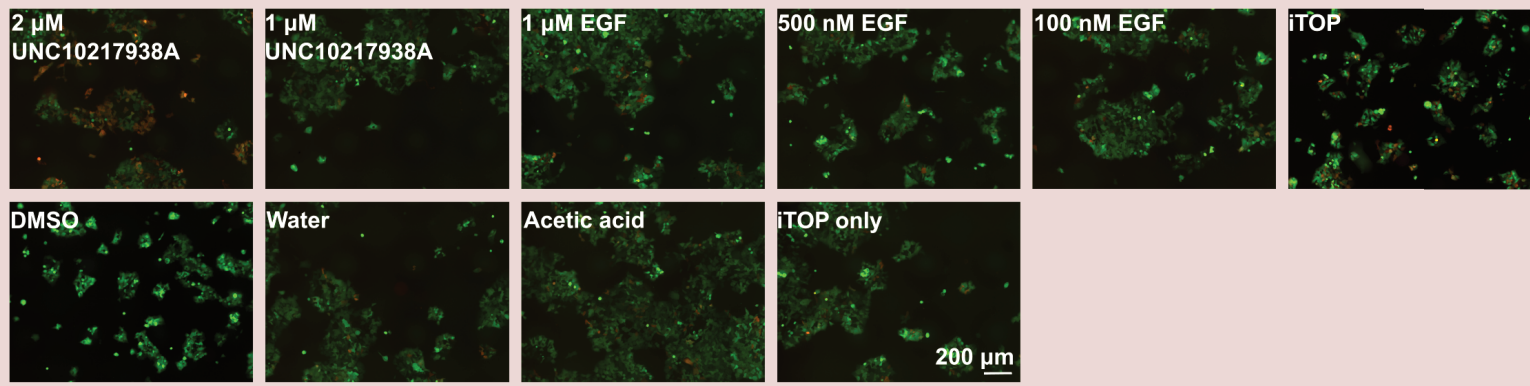

Figure 1. Chloroquine and UNC10217938A enhance TATCre functional delivery to recipient cells. (A) 5000 HEK293 Cre reporter cells were plated per well of a 384-well plate. Assuming a 24-h doubling time, $24 \mathrm{~h}$ later 10,000 cells were treated with or without $5.2 \mu \mathrm{g} / \mathrm{well}$ TATCre in the absence or presence of compounds that modify the uptake or escape of delivered cargoes. Cells were imaged for RFP fluorescence (a positive readout of Cre recombination) using IncuCyte ${ }^{\circledR}$ S3. The percentage of RFP positive cells from the total GFP positive cell population, normalized to a maximum of $100 \%$ is shown in the graph; $n=4$. Error bars \pm standard error of mean. Normally distributed data was analyzed using one way analysis of variance. Data, which were not normally distributed, were excluded from analysis of variance analysis and analyzed using a nonparametric unpaired t-test. Red line indicates baseline value for TATCre treatment of cells in the absence of uptake or endosomal escape enhancing compound. (B) Representative images associated with each treatment condition. Images exported from IncuCyte ${ }^{\circledR}$ S3 2018 software and arranged using Inkscape. Scale bar $200 \mu \mathrm{m} ; \mathrm{n}=4$.

Significance is indicated by colour coded asterisks that match statistically significant test conditions to the appropriate control. Asterisks in figure represent level of significance: ${ }^{*} p \leq 0.05, * * p \leq 0.01, * * * p \leq 0.001$ and ${ }^{* * * *} p \leq 0.0001$.

DMSO: Dimethyl sulfoxide; RFP: Red fluorescent protein. 


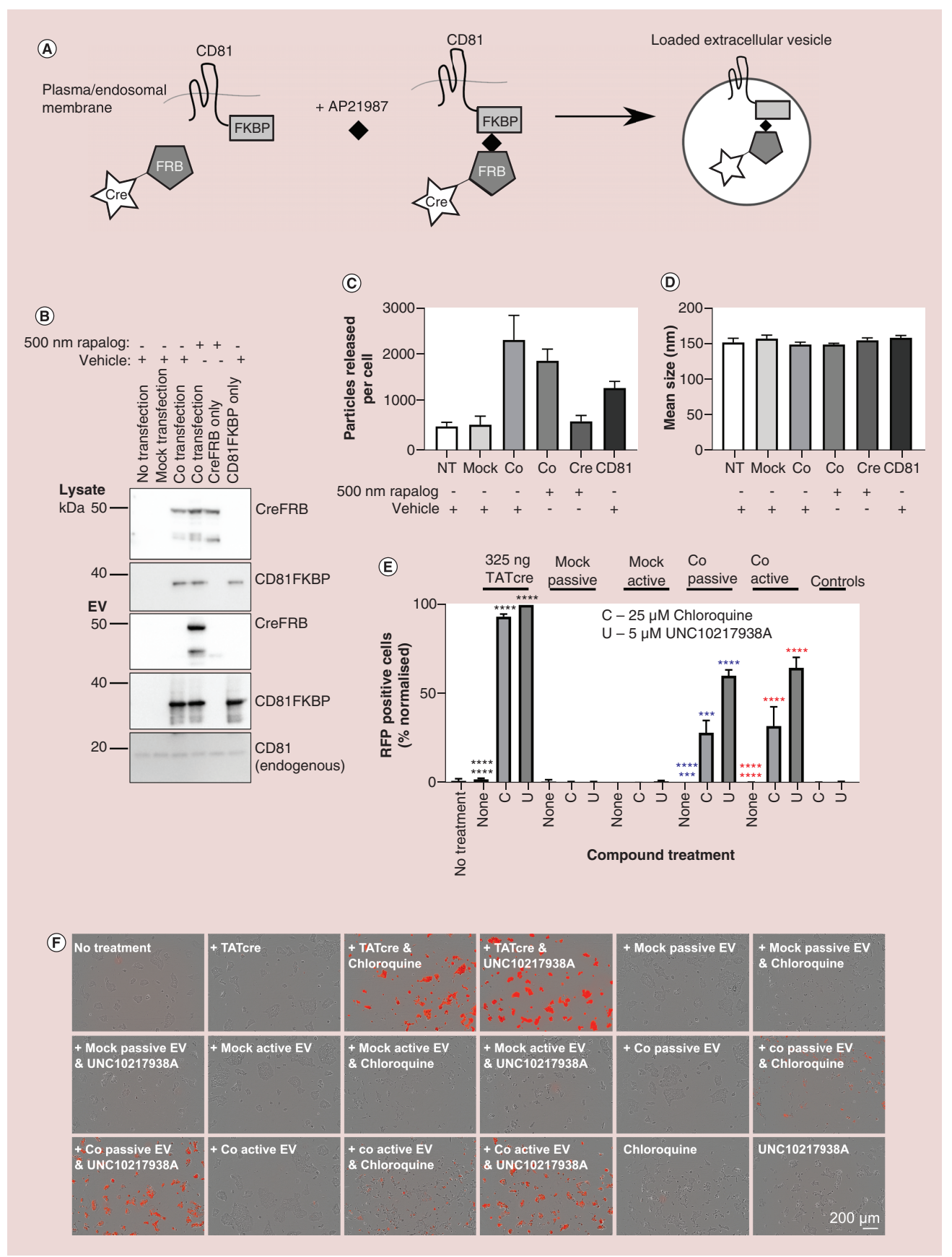

Figure 2. Rapalog-induced dimerization loads extracellular vesicles with CreFRB and functional delivery of CreFRB is only possible in the presence of endosomal escape enhancing compounds. (A) Diagram showing the rapalog-induced dimerization process. CreFRB dimerizes with EV marker CD81FKBP in the presence of rapalog AP21967. This means that CreFRB is in the vicinity of EV biogenesis and should be incorporated into EVs that are produced. (B) Representative western blots showing CreFRB and CD81FKBP expression in EV producing cell lysates and in the EVs isolated from transfected cells in the presence of $500 \mathrm{nM}$ rapalog or vehicle control. Cotransfection condition = both CreFRB and CD81FKBP were expressed in the cells otherwise cells were nontransfected, mock transfected or transfected individually with CreFRB or CD81FKBP. Total $40 \mu \mathrm{g} /$ well of lysate or equal volumes of EVs (corrected to cell number) were loaded onto the gel; $\mathrm{n}=3$. Details of antibodies used can be found in Supplementary Table 1. (C \& D) Nanoparticle tracking analysis was used to analyze the average number of particles released by each cell (C) and the size of EVs (D) $n=3$. Error bars \pm standard error of mean. (E) In a 384-well plate approximately 10,000 HEK293 Cre reporter cells were treated with either $325 \mathrm{ng}$ TATCre or $20 \mu \mathrm{g} / \mathrm{ml}(1 \mu \mathrm{g} / \mathrm{well}) \mathrm{EVs}$ isolated from mock transfected cells and from cells coexpressing both CreFRB and CD81FKBP (co) in the presence of ethanol vehicle control (passively loaded) or $31 \mathrm{nM}$ rapalog AP21967 (actively loaded). After $24 \mathrm{~h}$, the effect that $25 \mu \mathrm{M}$ chloroquine and $5 \mu \mathrm{M}$ UNC10217938A had upon functional delivery of EV associated CreFRB was assessed. The normalized percentage of RFP positive cells from the total GFP population is shown; $\mathrm{n}=3$. Error bars \pm standard error of mean. One way analysis of variance. (F) Images showing the RFP expression (positive readout for Cre recombination) for each condition as described in (E). Scale bar $200 \mu \mathrm{m}$. Images taken by IncuCyte ${ }^{\circledR}$ S3; $n=3$.

Significance is indicated by colour coded asterisks that match statistically significant test conditions to the appropriate control. Asterisks in figure represent level of significance: ${ }^{*} p \leq 0.05,{ }^{* *} p \leq 0.01, * * * p \leq 0.001$ and ${ }^{* * * *} p \leq 0.0001$

C: $25 \mu \mathrm{M}$ Chloroquine; EV: Extracellular vesicle; NT: No treatment; RFP: Red fluorescent protein; U: $5 \mu \mathrm{M}$ UNC10217938A. 
Figure 7 shows that Cre loading was still efficient at $31 \mathrm{nM}$ rapalog, although there was some experimental variability; we continued the rest of the experiments using $31 \mathrm{nM}$ rapalog.

Next, the ability of loaded EVs to deliver functional CreFRB to recipient cells was analyzed. In a 384-well plate, HEK293 Cre reporter cells were treated with $20 \mu \mathrm{g} / \mathrm{ml} \mathrm{EVs}(1 \mu \mathrm{g} /$ well/10,000 cells) and the percentage of RFP positive cells from the total population of GFP cells quantified. While EVs derived from mock transfected cells did not cause any Cre-recombination, EVs derived from CreFRB and CD81FKBP expressing cells could only deliver CreFRB in the presence of endosomal escape enhancing compounds chloroquine or UNC10217938A (Figure 2E $\& \mathrm{~F}$ ). This result indicates that the EVs are taken up by endocytosis but become trapped within the endosomal system decreasing the efficiency of cargo delivery. Most interestingly, we found that functional delivery of CreFRB via passively loaded and actively loaded CreFRB EVs was equally efficient (Figure $2 \mathrm{E} \& \mathrm{~F}$ ), indicating that active loading of proteins into EVs may not be necessary in this cell system. Simple overexpression may be sufficient to load EVs with the desired protein cargo.

\section{EVs passively loaded with CreFRB are sufficient for functional delivery}

We carried out an in-depth characterization of the EVs passively and actively loaded with CreFRB. Western blot showed that CD81FKBP was present in EVs isolated from both conditions, whereas CreFRB was loaded preferentially in the actively loaded EVs, although a faint signal for CreFRB was still observed in the passively loaded EVs (Figure 3A \& Supplementary Figure 8). All other EV markers (CD81, GAPDH, ALIX and TSG101) were present in equal quantities in both preparations. Calnexin as a marker for the presence of cellular debris in the EV preparations was also detectable in both conditions (Figure 3A). Cryo-electron microscope images revealed electron dense EVs with lipid bilayers, of the expected nanometer size range, intact and with minimal cell debris and protein aggregate contamination (Figure 3B). Furthermore, no difference in morphology or levels of protein contamination was observed in passively loaded EVs compared with actively loaded EVs.

To further understand the presence of calnexin in our EV preparations, and to confirm that CreFRB was being loaded into EVs and not solely secreted as a soluble protein, CreFRB loaded EVs were treated with trypsin and/or Triton X-100. Upon treatment of EVs with trypsin, calnexin and GAPDH signal was removed suggesting that GAPDH and calnexin EV preparation contamination was due to contaminating cell debris or secreted proteins co-pelleting with the EVs (Figure 3C \& Supplementary Figure 9). Trypsin treatment of Cre loaded EVs also decreased the EV marker CD81 signal as the antibody is raised against residues 90-210 of CD81, which spans the transmembrane (90-112 and 202-210) or extracellular (113-201) region of the protein meaning that the CD81 antibody epitope is likely to be degraded after exposure to trypsin. The signal for intravesicular EV markers ALIX, TSG101 and loaded cargo of CreFRB were decreased upon trypsin digestion, but only completely removed when EVs had been pretreated with $0.1 \%$ Triton X-100 detergent to disrupt their membrane integrity. This suggests that a proportion of detectable ALIX, TSG101 and CreFRB protein is loaded into EVs, while a proportion may be due to secreted proteins and cellular debris co-pelleting with EVs. The concerning presence of extra-vesicular proteins could be rectified by using engineered cells stably expressing the proteins of choice to avoid the drop in viability that transfection can cause. Alternatively, protein contamination of EV preparations could be reduced by using alternative isolation strategies that have been shown to remove contaminating proteins such as density gradients [52], size exclusion chromatography [53] and anion exchange chromatography [54].

Western blots of HEK293 Cre reporter cells treated with $20 \mu \mathrm{g} / \mathrm{ml}$ EVs in a six-well plate showed that CreFRB protein was present in recipient cells $24 \mathrm{~h}$ after treatment with EVs loaded by rapalog-induced dimerization, only when endosomal escape enhancers chloroquine and UNC10217938A were used (Figure 3D). Interestingly, the presence of the lower molecular weight band of Cre (cleavage by-product of CreFRB) was found in recipient cells treated with EVs that were both actively and passively loaded. These results indicate that in the absence of endosomal escape enhancers, CreFRB and Cre protein cleavage product remain within the endosomal system and are degraded in the lysosome within the $24 \mathrm{~h}$ treatment period. In the presence of escape compounds, the Cre/CreFRB cargo is able to escape the endosomal system and exert functional effects.

Since passively and actively loaded CreFRB EVs could deliver Cre recombinase activity to recipient cells with apparent equal efficiencies, we investigated whether this was due to oversaturation of cells with passively loaded CreFRB protein. Decreasing concentrations of TATCre was ran on western blots to make a standard curve from which unknown amounts of EV associated CreFRB could be calculated. The average number of CreFRB molecules per actively loaded EV was calculated to be 34.7 as compared with passively loaded EVs, which contained an average of 8.9 CreFRB molecules (Table $1 \&$ Supplementary Figure 10). This equated to the average concentration 


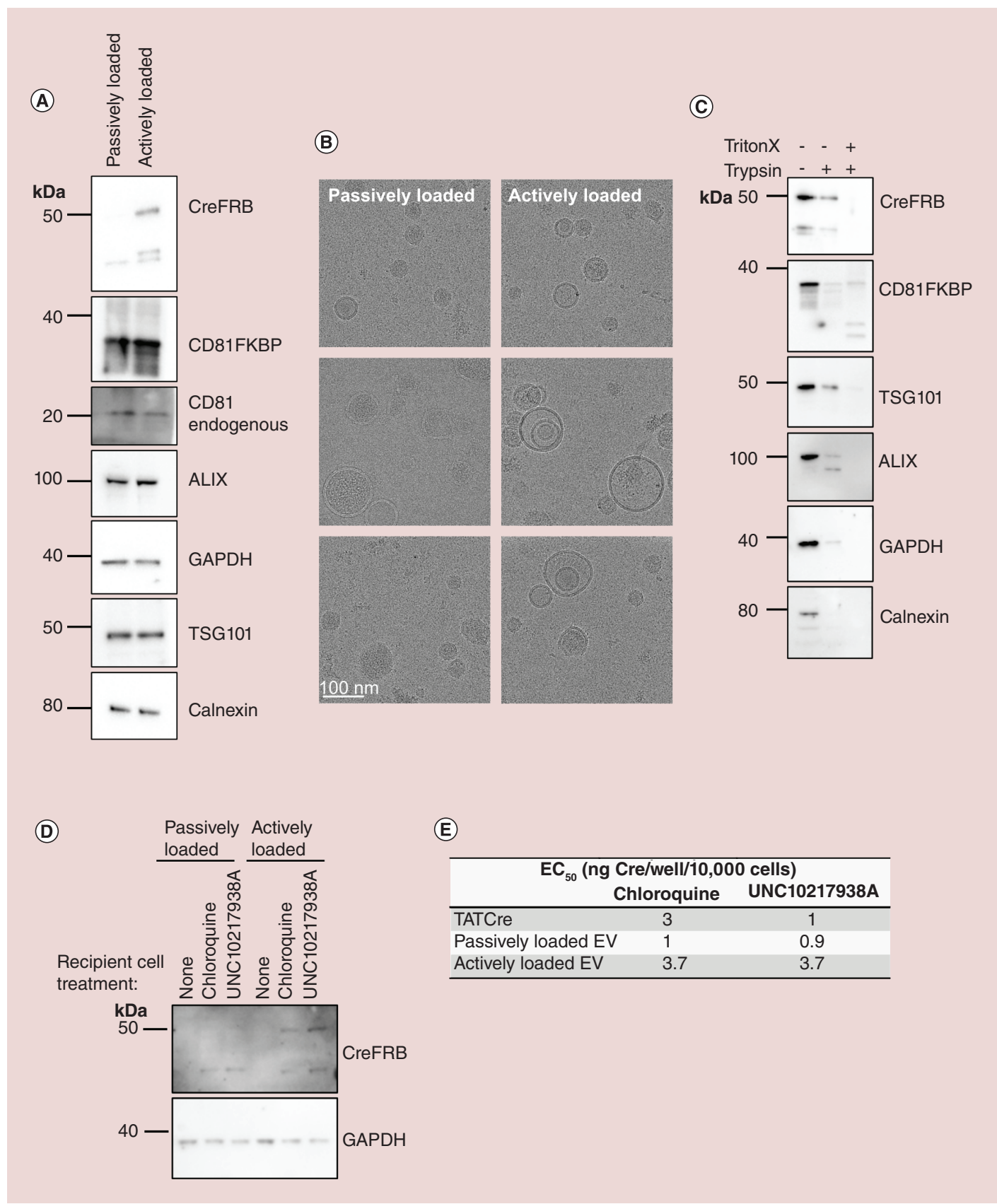

Figure 3. Active loading of CreFRB protein is not necessary for functional delivery; passive loading is sufficient. (A) EVs were isolated from CD81FKBP and CreFRB expressing cells in the presence of ethanol vehicle control (passively loaded) and or 31 nM AP21967 (actively loaded). EV preparation volume was corrected for cell number, and $7.5 \mu l$ of each EV preparation was loaded onto gels for western blotting of EV markers (CD81, ALIX and TSG101), ubiquitous marker GAPDH, non-EV marker (calnexin) and overexpressed CreFRB and CD81FKBP. One representative blot of $n=3$ experiments is shown. (B) Cryo electron microscopy images show the morphology and integrity of EVs preparations. Scale bar $100 \mathrm{~nm} ; \mathrm{n}=3$. (C) Total $10 \mu \mathrm{g}$ EVs were treated with $0.0008 \% / 3 \mu \mathrm{M}$ trypsin $+/-0.1 \%$ Triton X-100, or left untreated. Resultant EVs were analyzed by western blot for CreFRB, CD81FKBP and other EV markers (TSG101 and ALIX), ubiquitous marker GAPDH and contaminant marker calnexin; $n=3$. (D) HEK293 Cre reporter cells were plated into a six-well plate and $24 \mathrm{~h}$ later media was refreshed to media containing $20 \mu \mathrm{g} / \mathrm{ml}$ EVs that were either passively loaded or actively loaded with CreFRB in the presence and absence of $25 \mu \mathrm{M}$ chloroquine and $5 \mu \mathrm{M}$

UNC10217938A. After a $24 \mathrm{~h}$ incubation, cells were lysed and equal volumes of lysate from each condition western blotted for the presence of CreFRB protein; $n=3$. (E) Approximately 10,000 HEK293 Cre reporter cells/well were treated with decreasing concentrations of CreFRB actively loaded EVs from $1 \mu \mathrm{g} /$ well -0 (average of $19.22 \mathrm{ng}-0$ CreFRB/well as calculated in Table 1), passively loaded EV from $1 \mu \mathrm{g} /$ well -0 (5.63 ng -0 CreFRB/well as calculated in Table 1) and TATCre (20-0 ng/well). The efficiency of functional delivery, in the presence and absence of endosomal escape enhancing compounds $25 \mu \mathrm{M}$ chloroquine and $5 \mu \mathrm{M}$ UNC10217938A, was analyzed by quantifying percentage of RFP positive cells $24 \mathrm{~h}$ after treatment started. From this data, the $\mathrm{EC}_{50}$ for each EV condition was calculated using GraphPad Prism; $\mathrm{n}=3$. Error bars \pm standard error of mean. 
Table 1. Calculation of CreFRB extracellular vesicle content.

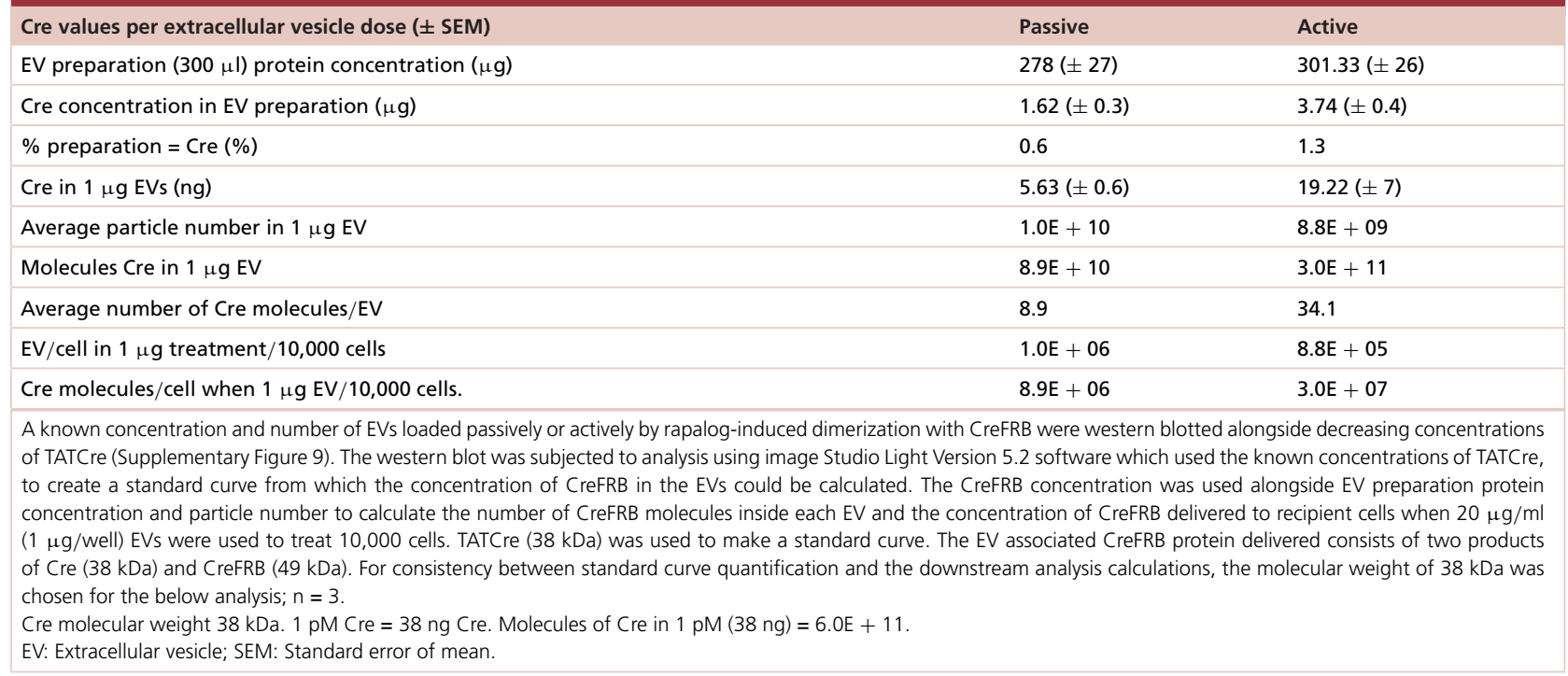

of CreFRB per $1 \mu \mathrm{g}$ EV treatment of 10,000 cells to be $19.2 \mathrm{ng}$ (3E7 molecules of Cre/cell) upon active loading, or 5.65 ng CreFRB (8.9E6 molecules Cre/cell) when EVs are loaded passively. This indicated that the oversaturation hypothesis should be investigated and that differences in functional delivery efficiencies of the EVs may become apparent in a dose-response curve and calculation of the $\mathrm{EC}_{50}$.

Approximately 10,000 cells in a 384-well plate were treated with decreasing concentrations of EVs $0-1 \mu \mathrm{g}-$ in the presence and absence of chloroquine and UNC10217938A to calculate the $\mathrm{EC}_{50}$ of CreFRB delivered by EVs in each condition. $1 \mu \mathrm{g} \mathrm{EV}$ equated to 5.65-0 ng cre for passively loaded EVs and 19.2-0 ng cre for actively loaded EVs. Remarkably, the $\mathrm{EC}_{50}$ of passively loaded EVs was $1 \mathrm{ng}$ Cre/10,000 cells and 0.9 ng Cre/10,000 cells in the presence of chloroquine and UNC10217938A respectively, as compared with EVs actively loaded with CreFRB which needed $3.7 \mathrm{ng}$ CreFRB/10,000 cells in the presence of both compounds to achieve 50\% response (Figure 3E \& Supplementary Figures 11B \& C). Active loading of CreFRB protein was not necessary for the functional delivery of CreFRB via EVs and passively loaded EVs were actually more efficient at delivering CreFRB. Interestingly, although not comparable to EV delivery of CreFRB, the $\mathrm{EC}_{50}$ showed that TATCre delivery (20-0 ng) was less efficient in the presence of chloroquine (EC50 TATCre $3 \mathrm{ng}$ ) as compared to UNC10217938A (EC50 $1 \mathrm{ng}$ ) (Figure 3E \& Supplementary Figure 11A).

These data indicate that passive loading of EVs with CreFRB in Expi293 $\mathrm{F}^{\mathrm{TM}}$ cells by overexpression is sufficient for functional delivery to recipient cells in the presence of endosomal escape enhancing compounds. However, the possibility of extravesicular CreFRB protein or nucleic acid being the delivered factor cannot be excluded.

\section{Intravesicular loaded CreFRB is essential for functional delivery}

To exclude the possibility that extra-vesicular nucleic acids such as mRNA/plasmid DNA remaining from transfection were being delivered to recipient cells alongside EVs, EV preparations were treated with a nuclease which digests both double stranded and single stranded nucleic acids. A total of $100 \mu \mathrm{g}$ plasmid DNA can be completely digested within $30 \mathrm{~min}$ at $37^{\circ} \mathrm{C}$ (Supplementary Figure 12). Nuclease treatment of EVs did not alter the efficiency of functional delivery of CreFRB to recipient cells (Figure $4 \mathrm{~A} \& \mathrm{~B}$ ). This indicated that if nucleic acids were contributing to CreFRB functional delivery, then mRNA must be enclosed within the EVs. These results also indicate that no residual DNA from transfection was being transferred to recipient cells, as any remaining DNA remaining, would have been extra-vesicular and digested by the nuclease.

To determine whether the cargo delivered to recipient cells was inside the EVs or soluble contaminating protein pelleted with EVs, $20 \mu \mathrm{g} / \mathrm{ml} \mathrm{EVs} \mathrm{that} \mathrm{were} \mathrm{passively} \mathrm{or} \mathrm{actively} \mathrm{loaded} \mathrm{with} \mathrm{CreFRB,} \mathrm{were} \mathrm{treated} \mathrm{with} 0.1 \%$ Triton X-100 for 30 min before being used to treat HEK293 Cre reporter cells with $1 \mu \mathrm{g} \mathrm{EV/well/10,000} \mathrm{cells}$ for $24 \mathrm{~h}$ in a 384-well plate. The EVs that had not been lysed could deliver functional CreFRB to recipient cells; however, after lysis, EVs were unable to sufficiently deliver functional CreFRB (Figure 4C). On the other hand, 


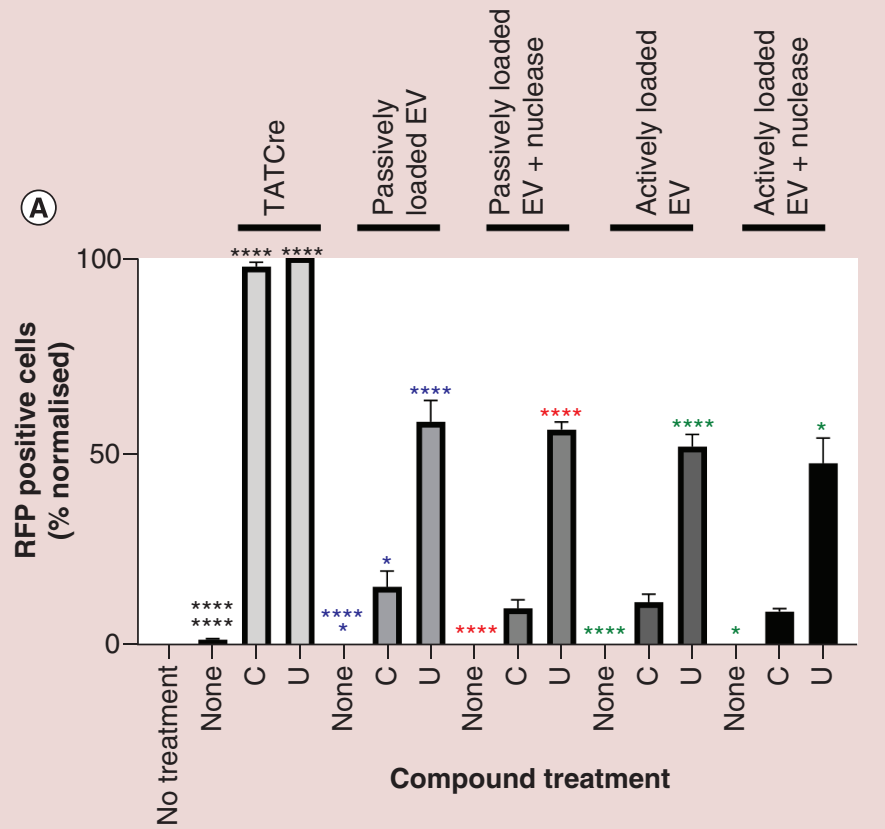

(B) Compound treatment
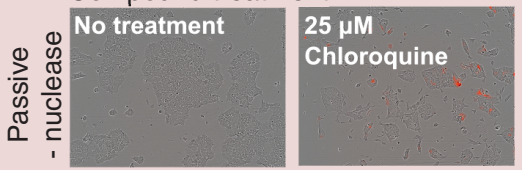

$5 \mu \mathrm{M}$

UNC10217938A
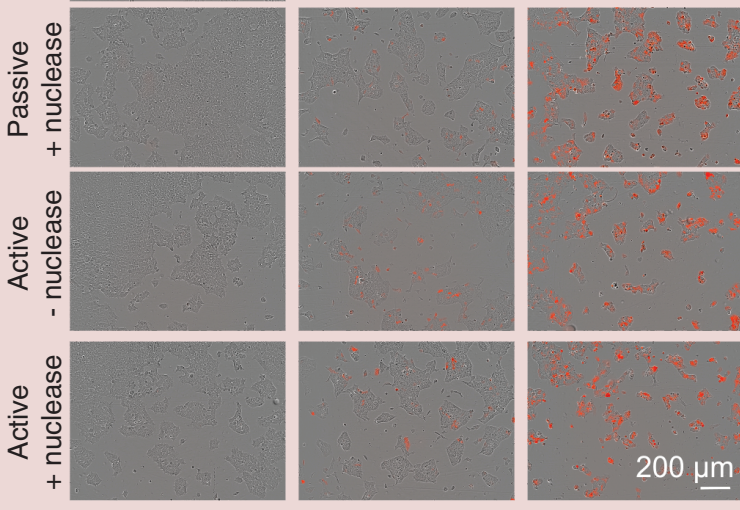

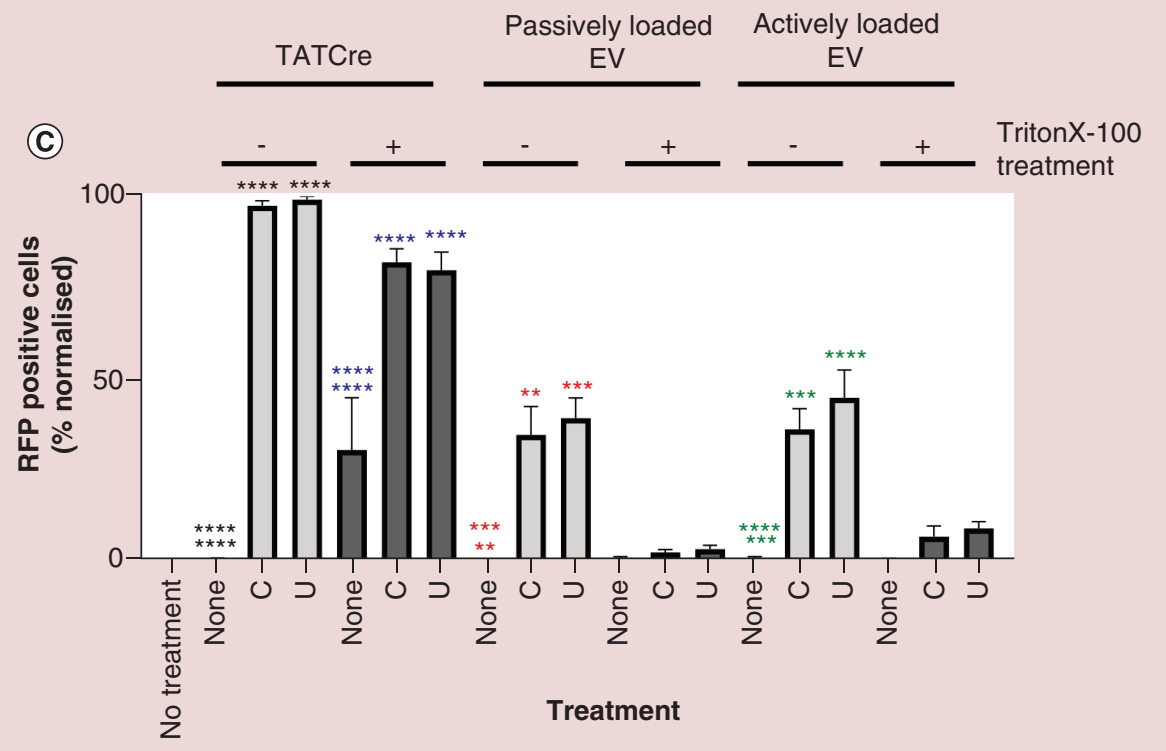

(D)

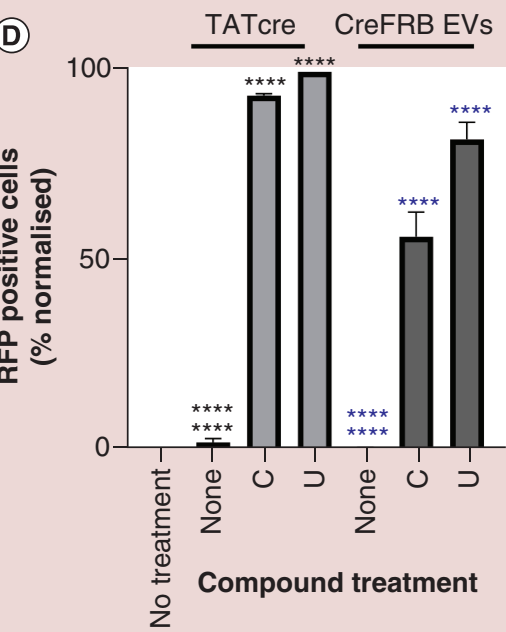

Figure 4. Intravesicular loaded CreFRB is essential for functional delivery, extra-vesicular contaminating nucleic acids and protein have no role in functional delivery. (A) $20 \mu \mathrm{g}$ EVs were treated with the nuclease Basemuncher $(1: 10,000)$ in phosphate-buffered solution supplemented with $2 \mathrm{mM} \mathrm{MgCl}$. Excess nuclease was removed by pelleting the EVs by ultracentrifugation at 100,000 g. Recipient HEK293 Cre reporter cells were then treated with $20 \mu \mathrm{g} / \mathrm{ml}(1 \mu \mathrm{g} /$ well $)$ EVs with or without $25 \mu \mathrm{M}$ chloroquine or $5 \mu \mathrm{M}$ UNC10217938A. The percentage of GFP expressing cells that also expressed RFP normalized to a maximum of $100 \%$ was then calculated from the images acquired by IncuCyte ${ }^{\circledR} \mathrm{S} 3$ after 24-h treatment; $\mathrm{n}=3$, error bars \pm standard error of mean. One way analysis of variance was used for normally distributed data. Data, which were not normally distributed, were excluded from analysis of variance analysis and analysed using a nonparametric unpaired t-test. (B) Representative images for (A) after $24 \mathrm{~h}$ EV treatment. (C) TATCre and passively/actively loaded EVs were treated with or without $0.1 \%$ Triton X-100 for $30 \mathrm{~min}$ at room temperature before $325 \mathrm{ng}$ TATCre/well and $20 \mu \mathrm{g} / \mathrm{ml} \mathrm{EV}$ $(1 \mu \mathrm{g} /$ well) were added to HEK293 Cre reporter cells with or without $25 \mu \mathrm{M}$ chloroquine and $5 \mu \mathrm{M}$ UNC10217938A. The efficiency of CreFRB delivery was quantified by analyzing the percentage of RFP positive cells $24 \mathrm{~h}$ after treatment; $\mathrm{n}=3$, error bars \pm standard error of mean. (D) EVs were isolated from Expi293F ${ }^{\mathrm{TM}}$ cells overexpressing CreFRB only. HEK293 Cre reporter cells were treated with $20 \mu \mathrm{g} / \mathrm{ml}$ $(1 \mu \mathrm{g} /$ well) EVs or $325 \mathrm{ng}$ TATCre, and functional delivery was assessed by quantifying the percentage of cells expressing RFP normalized to $100 \%$ after a 24 -h treatment; $n=3$, error bars \pm standard error of mean. One-way analysis of variance.

Significance is indicated by colour coded asterisks that match statistically significant test conditions to the appropriate control. Asterisks in figure represent level of significance: ${ }^{*} p \leq 0.05, * * p \leq 0.01, * * * p \leq 0.001$ and $* * * * p \leq 0.0001$.

C: $25 \mu \mathrm{M}$ Chloroquine; EV: Extracellular vesicle; RFP: Red fluorescent protein; U: $5 \mu \mathrm{M}$ UNC10217938A. 
TATCre functional delivery was not negatively affected by Triton X-100 treatment. This suggests that the CreFRB cargo that was being delivered by EVs was within the EV lumen, and secreted protein contaminants of the EV pellet were not involved. The possibility that mRNA encoding CreFRB may be present in EVs and delivered to recipient cells alongside protein CreFRB protein cargoes cannot be ruled out. Finally, we further streamlined our system to overexpress CreFRB protein alone in Expi293 $\mathrm{F}^{\mathrm{TM}}$ cells for passive loading into EVs. The EVs isolated from transfected cells were used to treat 10,000 cells in 384-well plate. The functional delivery of EV associated CreFRB to recipient cells was found to be efficient using the streamlined EVs in the presence of chloroquine and UNC10217938A (Figure 4D).

\section{Discussion}

In this study, we have identified two endosomal escape enhancing compounds, chloroquine and UNC10217938A, which could be used in conjunction with EVs to enhance functional delivery of EV cargoes. Primarily, we carried out a small compound screen to identify compounds that aid functional delivery of TATCre to recipient cells. EVs can be time consuming to isolate, and large amounts of EVs would be necessary for a compound screen. Therefore, we assessed efficiency of the compounds using a more accessible reagent - recombinant TATCre protein. While EVs are known to be taken up into cells by several mechanisms including endocytosis, unmodified proteins are less efficiently taken up into cells. The inclusion of the basic cell penetrating peptide TAT sequence in the Cre recombinase protein sequence is commonly used to increase cell penetration and functional delivery of Cre recombinase [55]. The uptake mechanism of TAT cell penetrating peptides is widely debated but is thought to be in part, like EVs, via endocytosis making them interesting candidates for the study of cellular uptake mechanisms [56].

The experiment revealed some interesting information regarding the mechanism of TATCre delivery to recipient cells. AmphotericinB enhanced functional delivery of TATCre by forming pores in the plasma membrane. Macropinocytosis and macropinosome escape enhancer iTOP solution [47] increased TATCre delivery and recombinase activity in recipient cells. However, EGF did not significantly improve the functional delivery of TATCre suggesting that expression of K-RAS and/or serum starvation may be necessary in conjunction with EGF treatment to have an impact upon macropinocytosis of EVs [35]. Lysosomotropic endosomal escape enhancing agents chloroquine and amantadine were effective at enhancing TATCre delivery, as was UNC10217938A. The mechanism of action of UNC10217938A is not well understood. It is believed to enhance endosomal escape of ASOs in a nonlysosomotropic mechanism after interaction with specific targets within the endosomal system [46]. These results indicate that TATCre can be taken up into cells in several ways; passage though membrane pores, macropinocytosis and endocytosis.

Interestingly, our results show that the compounds that enhance TATCre delivery (chloroquine and UNC10217938A) also promote functional delivery of loaded EV CreFRB cargo to recipient cells suggesting that EVs and TATCre have similar mechanisms of uptake. There is growing interest in using EVs as therapeutic delivery vehicles and so identifying compounds that will make delivery of EV cargo more efficient is important. As EVs can be time consuming to isolate, TATCre could be used in place of EVs for larger scale compound screening for novel EV delivery enhancing compounds.

When an EV is endocytosed, it is thought to remain intact within the endosome. If this is the case, the EV would need to either fuse with the endosomal membrane or be destroyed after endosomal escape in order to release their cargoes [39]. Our results indicate that endosomal escape of EVs is essential for functional delivery of cargo suggesting that fusion with the endosomal membrane does not take place. Lysosomotropic compounds cause changes in osmolarity within the endosome lumen, which would consequently impact the osmolarity of residing EVs leading to their destruction and release of cargoes for functional delivery [43].

We investigated rapalog-induced dimerization of FKBP and FRB fusion proteins to load EVs with CreFRB. Surprisingly, we found that passive loading of EVs was sufficient to deliver functional cargo to recipient cells in the presence of endosomal escape enhancing compounds. The EVs actively loaded with CreFRB had a higher $\mathrm{EC}_{50}$ for the concentration of CreFRB necessary to be delivered to achieve a $50 \%$ response, as compared with passively loaded EVs. The decreased efficiency of actively loaded EVs could be due to inefficient dissociation of CD81FKBP away from CreFRB cargo. The dissociation constant $\left(\mathrm{K}_{\mathrm{d}}\right)$ between FRB and the rapalog-FKBP complex has been shown to be $12 \mathrm{nM}$ assuming dynamic equilibrium [30]. It would be expected that upon rapalog dilution or in the absence of the rapalog, a new stable equilibrium would form allowing the heterodimerization complex to dissociate. Supporting this, others have successfully achieved nanoparticle loading and functional delivery of nuclear proteins 
using this heterodimerization system [33]. However, our results indicate that dissociation of the CreFRB cargo away from CD81FKBP can be inefficient.

VLPs, called gesicles, can be actively loaded with Cas9 by rapalog-induced dimerization. Gesicles can deliver their functional cargoes efficiently in the absence of endosomal escape enhancing compounds in vitro [33]. This may be because VSV-g expression in the gesicle membrane acts as a cell-penetrating peptide to aid VLP uptake and subsequently facilitates endosomal escape of the VLPs upon changes in $\mathrm{pH}$ within the endosomal system [57-59]. The presence of viral proteins in gesicles may cause an immunogenic response, increased rates of clearance and restrict the potential of using repeated doses [60]. The advantages and risks of using immunogenic viral glycoproteins or other cell-penetrating peptides versus potentially toxic endosomal escape compounds for clinical applications needs to be assessed.

An additional study used rapalog-induced dimerization to load EVs with a transcription factor. Contrasting with our results, functional delivery was observed using loaded EV [61] without the aid of any endosomal escape enhancers. There could be multiple explanations for these differing observations; the transcription factor being delivered by Lainscek et al. may get packaged into EVs more efficiently. Additionally, the amount of protein that needs to be functionally delivered to observe an effect may be different to CreFRB. Where we observed that simple passive loading of EVs by overexpression is sufficient to deliver functional cargoes in the presence of endosomal escape inhibitors, Lainscek et al. found that active loading of the transcription factor using rapalog dimerization was essential for functional delivery to be achieved. This could be attributed to the Expi293 $\mathrm{F}^{\mathrm{TM}}$ cells that are adapted for high mRNA and protein expression, which may drive passive loading of EVs with overexpressed cargoes.

Overall, Expi293 $\mathrm{F}^{\mathrm{TM}}$ cells can be cultured at high densities in large volumes, which can be handled easily, are nontoxic and nonimmunogenic in vivo [18,19]. Additionally, simple overexpression of a protein can result in loading of it as cargo into EVs, which can be delivered in a functional capacity in the presence of endosomal escape enhancing compounds. These points make Expi293 $\mathrm{F}^{\mathrm{TM}}$ cells an interesting choice for clinical EV therapeutic applications. However, the use of very toxic endosomal escape agents such as chloroquine is potentially not an option in vivo at the doses which facilitate endosomal escape. To avoid systemic injection and the related toxicities, EVs engineered to incorporate endosomal escape enhancing compounds into their lipid bilayer could be targeted to specific tissues of interest, where after uptake, the compound would be released locally to aid endosomal escape avoiding systemic toxicity issues. The UNC10217938A compound, however, is still relatively uninvestigated regarding its tolerance, potency and toxicity in vivo. Although UNC10217938A has been shown to be useful and efficient at enhancing endosomal escape of ASOs [46], this is the first study demonstrating its usefulness in facilitation endosomal escape and functional delivery of EV cargos. UNC10217938A was used in vivo to aid delivery of ASOs and no significant toxicity was observed when used at $7.5 \mathrm{mg} / \mathrm{kg}$ [46]. If further work confirms UNC10217938A is well tolerated with low toxicity in vivo, it could be a promising compound to use systemically or in conjunction with EV therapeutics. An in-depth analysis of the safety profile of this compound could address issues such as in vivo toxicity and allow us to further the findings presented in this study to investigate in vivo delivery of passively loaded EV cargoes in the presence of endosomal escape enhancing compounds.

\section{Conclusion \& future perspective}

Our study raises exciting possibilities for the use of EVs in therapeutic applications. Delivery of functional biological cargoes to recipient cells is a difficult task, but if successful could hold endless possibilities for treatments of a broad range of diseases. For successful delivery, therapeutic cargoes need to be protected from degradation, protected from clearance by an immune response and delivered in a functional capacity. These are inherent characteristics of EVs which make them ideal candidates for the task. A common bottle neck of using nano-carriers to deliver therapeutic cargoes is their subsequent entrapment within the endosomal system after endocytosis into the recipient cell. Our study indicates that endosomal escape enhancing compounds could be used in conjunction with EV therapeutics to enhance functional delivery of their cargoes. Further development of EV-endosomal escape enhancing compound formulations could see EVs become the next generation of nano-therapeutic agents. 
Author contributions

N Heath acquired and analyzed data. X Osteikoetxea carried out EV treatment with triton X-100 experiments and analyzed their functional delivery. TM de Oliveria carried out cryo electron microscopy image acquisition. N Tigue, LM Mayr, R Overman, N Dekker and R Davies conceptualized and supervised the project. N Heath, E Lázaro-lbáñez, O Shatnyeva, X Osteikoetxea and C Schindler participated in idea formulation and experimental design. $\mathrm{N}$ Heath wrote manuscript and made figures. All authors reviewed and edited manuscript.

\section{Acknowledgments}

Authors would like to thank Blueberry Therapeutics for use of their NanoSight for nanoparticle tracking analysis. We additionally acknowledge the ThermoFisher-Pharma CryoEM consortium (Cambridge, UK) for the use of their facilities for imaging

\section{Financial \& competing interests disclosure}

The work was fully funded by AstraZeneca. All authors are current or former salaried employees of AstraZeneca and have/had stock or stock options in AstraZeneca. N Heath, E Lázaro-lbáñez, O Shatnyeva and X Osteikoetxea are/were fellows of the AstraZeneca postdoc program. The authors have no other relevant affiliations or financial involvement with any organization or entity with financial interest in or financial conflict with the subject matter or materials discussed in the manuscript apart from those disclosed.

No writing assistance was utilized in the production of this manuscript.

\section{Open access}

This work is licensed under the Attribution-NonCommercial-NoDerivatives 4.0 Unported License. To view a copy of this license, visit http://creativecommons.org/licenses/by-nc-nd/4.0/

\section{Summary points}

- Functional delivery of TATCre is increased by uptake enhancing and endosomal escape enhancing compounds.

- CreFRB protein is actively loaded into extracellular vesicles (EVs) using rapalog-induced dimerization to CD81FKBP and passively loaded into EVs by overexpression.

- Functional delivery of EV associated CreFRB is only possible in the presence of the endosomal escape enhancing compounds chloroquine and UNC10217938A.

- Passive loading of CreFRB by overexpression is sufficient for functional delivery of CreFRB to recipient cells in the presence of chloroquine and UNC10217938A, active loading of CreFRB provides no further benefit.

- Passively loaded EVs could be used in conjunction with endosomal escape enhancing compounds to deliver therapeutic cargoes in future clinical applications.

\section{References}

Papers of special note have been highlighted as: $\bullet$ of interest; $\bullet \bullet$ of considerable interest

1. Allen TM, Cullis PR. Liposomal drug delivery systems: from concept to clinical applications. Adv. Drug Deliv. Rev. 65(1), 36-48 (2013).

2. Ilinskaya AN, Dobrovolskaia MA. Understanding the immunogenicity and antigenicity of nanomaterials: past, present and future. Toxicol. Appl. Pharmacol. 299, 70-77 (2016).

3. Tatiparti K, Sau S, Kashaw SK, Iyer AK. siRNA delivery strategies: a comprehensive review of recent developments. Nanomaterials (Basel) 7(4), (2017).

4. Zuris JA, Thompson DB, Shu Y et al. Cationic lipid-mediated delivery of proteins enables efficient protein-based genome editing in vitro and in vivo. Nat. Biotechnol. 33(1), 73-80 (2015).

5. Bozzuto G, Molinari A. Liposomes as nanomedical devices. Int. J. Nanomedicine 10, 975-999 (2015).

6. Fais S, O'Driscoll L, Borras FE et al. Evidence-based clinical use of nanoscale extracellular vesicles in nanomedicine. ACS Nano 10(4), 3886-3899 (2016).

7. Stremersch S, De Smedt SC, Raemdonck K. Therapeutic and diagnostic applications of extracellular vesicles. J. Control. Release $244(\mathrm{Pt}$ B), 167-183 (2016).

8. Raposo G, Stoorvogel W. Extracellular vesicles: exosomes, microvesicles, and friends. J. Cell Biol. 200(4), 373-383 (2013).

9. Van Niel G, D’Angelo G, Raposo G. Shedding light on the cell biology of extracellular vesicles. Nat. Rev. Mol. Cell Biol. 19(4), 213-228 (2018).

10. Yanez-Mo M, Siljander PR, Andreu Z et al. Biological properties of extracellular vesicles and their physiological functions. J Extracell. Vesicles 4, 27066 (2015). 
11. Colombo M, Raposo G, Thery C. Biogenesis, secretion, and intercellular interactions of exosomes and other extracellular vesicles. Annu. Rev. Cell Dev. Biol. 30, 255-289 (2014).

12. Valadi H, Ekstrom K, Bossios A, Sjostrand M, Lee JJ, Lotvall JO. Exosome-mediated transfer of mRNAs and microRNAs is a novel mechanism of genetic exchange between cells. Nat. Cell Biol. 9(6), 654-659 (2007).

13. Pegtel DM, Cosmopoulos K, Thorley-Lawson DA et al. Functional delivery of viral miRNAs via exosomes. Proc. Natl Acad. Sci. USA 107(14), 6328-6333 (2010).

14. Luga V, Zhang L, Viloria-Petit AM et al. Exosomes mediate stromal mobilization of autocrine Wnt-PCP signaling in breast cancer cell migration. Cell 151(7), 1542-1556 (2012).

15. Fevrier B, Vilette D, Archer F et al. Cells release prions in association with exosomes. Proc. Natl Acad. Sci. USA 101(26), 9683-9688 (2004).

16. Rajendran L, Honsho M, Zahn TR et al. Alzheimer's disease beta-amyloid peptides are released in association with exosomes. Proc. Natl Acad. Sci. USA 103(30), 11172-11177 (2006).

17. Stahl PD, Raposo G. Exosomes and extracellular vesicles: the path forward. Essays Biochem. 62(2), 119-124 (2018).

18. Zhu X, Badawi M, Pomeroy $S$ et al. Comprehensive toxicity and immunogenicity studies reveal minimal effects in mice following sustained dosing of extracellular vesicles derived from HEK293T cells. J. Extracell. Vesicles 6(1), 1324730 (2017).

- This study provides proof of concept that cell line derived extracellular vesicles (EVs) could be used as a therapeutic delivery vehicle as they do not induce toxicity or immunogenicity in vitro or in vivo.

19. Saleh AF, Lazaro-Ibanez E, Forsgard MA et al. Extracellular vesicles induce minimal hepatotoxicity and immunogenicity. Nanoscale 11(14), 6990-7001 (2019).

-. This study provides proof of concept that HEK derived cell line EXPI293F ${ }^{T M}$ EVs do not induce toxicity or immunogenicity in vitro or in vivo making them an interesting cell line candidate for use in EV therapeutics.

20. Kamerkar $\mathrm{S}$, Lebleu VS, Sugimoto $\mathrm{H}$ et al. Exosomes facilitate therapeutic targeting of oncogenic KRAS in pancreatic cancer. Nature 546(7659), 498-503 (2017).

21. Kim MS, Haney MJ, Zhao Y et al. Development of exosome-encapsulated Paclitaxel to overcome MDR in cancer cells. Nanomedicine 12(3), 655-664 (2016).

22. Tian Y, Li S, Song J et al. A doxorubicin delivery platform using engineered natural membrane vesicle exosomes for targeted tumor therapy. Biomaterials 35(7), 2383-2390 (2014).

23. Hadla M, Palazzolo S, Corona G et al. Exosomes increase the therapeutic index of doxorubicin in breast and ovarian cancer mouse models. Nanomedicine (Lond.) 11(18), 2431-2441 (2016).

24. Saari H, Lazaro-Ibanez E, Viitala T, Vuorimaa-Laukkanen E, Siljander P, Yliperttula M. Microvesicle- and exosome-mediated drug delivery enhances the cytotoxicity of Paclitaxel in autologous prostate cancer cells. J. Control. Release 220(Pt B), 727-737 (2015).

25. Lin Y, Wu J, Gu W et al. Exosome-liposome hybrid nanoparticles deliver CRISPR/Cas9 system in MSCs. Adv. Sci. (Weinh.) 5(4), 1700611 (2018).

26. Alvarez-Erviti L, Seow Y, Yin H, Betts C, Lakhal S, Wood MJ. Delivery of siRNA to the mouse brain by systemic injection of targeted exosomes. Nat. Biotechnol. 29(4), 341-345 (2011).

- This study shows that EVs can deliver functional nucleic acids in vivo.

27. Kojima R, Bojar D, Rizzi G et al. Designer exosomes produced by implanted cells intracerebrally deliver therapeutic cargo for Parkinson's disease treatment. Nat. Commun. 9(1), 1305 (2018).

28. Sterzenbach U, Putz U, Low LH, Silke J, Tan SS, Howitt J. Engineered exosomes as vehicles for biologically active proteins. Mol. Ther. 25(6), 1269-1278 (2017).

29. Yim N, Ryu SW, Choi K et al. Exosome engineering for efficient intracellular delivery of soluble proteins using optically reversible protein-protein interaction module. Nat. Commun. 7, 12277 (2016).

- This study shows that light induced dimerisation could be used to load EVs with protein cargoes that were delivered in a functional capacity to recipient cells.

30. Banaszynski LA, Liu CW, Wandless TJ. Characterization of the FKBP.rapamycin.FRB ternary complex. J. Am. Chem. Soc. 127(13), 4715-4721 (2005).

31. Graef IA, Holsinger LJ, Diver S, Schreiber SL, Crabtree GR. Proximity and orientation underlie signaling by the non-receptor tyrosine kinase ZAP70. EMBO J. 16(18), 5618-5628 (1997).

32. Castellano F, Montcourrier P, Guillemot JC et al. Inducible recruitment of Cdc42 or WASP to a cell-surface receptor triggers actin polymerization and filopodium formation. Curr. Biol. 9(7), 351-360 (1999).

33. Campbell LA, Coke LM, Richie CT, Fortuno LV, Park AY, Harvey BK. Gesicle-mediated delivery of CRISPR/Cas9 ribonucleoprotein complex for inactivating the HIV provirus. Mol. Ther. 27(1), 151-163 (2019).

-• This study used rapalog induced dimerisation to load virus like particles called gesicles with gene editing machinery. 
34. Del Conde I, Shrimpton CN, Thiagarajan P, Lopez JA. Tissue-factor-bearing microvesicles arise from lipid rafts and fuse with activated platelets to initiate coagulation. Blood 106(5), 1604-1611 (2005).

35. Nakase I, Kobayashi NB, Takatani-Nakase T, Yoshida T. Active macropinocytosis induction by stimulation of epidermal growth factor receptor and oncogenic Ras expression potentiates cellular uptake efficacy of exosomes. Sci. Rep. 5, 10300 (2015).

36. Tian T, Zhu YL, Zhou YY et al. Exosome uptake through clathrin-mediated endocytosis and macropinocytosis and mediating miR-21 delivery. J. Biol. Chem. 289(32), 22258-22267 (2014).

37. McKelvey KJ, Powell KL, Ashton AW, Morris JM, McCracken SA. Exosomes: mechanisms of uptake. J. Circ. Biomark 4, 7 (2015).

38. Mulcahy LA, Pink RC, Carter DR. Routes and mechanisms of extracellular vesicle uptake. J. Extracell. Vesicles 3, (2014).

39. Mathieu M, Martin-Jaular L, Lavieu G, Thery C. Specificities of secretion and uptake of exosomes and other extracellular vesicles for cell-to-cell communication. Nat. Cell Biol. 21(1), 9-17 (2019).

40. Heusermann W, Hean J, Trojer D et al. Exosomes surf on filopodia to enter cells at endocytic hot spots, traffic within endosomes, and are targeted to the ER. J. Cell Biol. 213(2), 173-184 (2016).

41. Andreoli TE. The structure and function of amphotericin B-cholesterol pores in lipid bilayer membranes. Ann. N. Y. Acad. Sci. 235(0), 448-468 (1974).

42. Lonn P, Kacsinta AD, Cui XS et al. Enhancing endosomal escape for intracellular delivery of macromolecular biologic therapeutics. Sci. Rep. 6, 32301 (2016).

43. Varkouhi AK, Scholte M, Storm G, Haisma HJ. Endosomal escape pathways for delivery of biologicals. J. Control. Release 151(3), 220-228 (2011).

44. Fredericksen BL, Wei BL, Yao J, Luo T, Garcia JV. Inhibition of endosomal/lysosomal degradation increases the infectivity of human immunodeficiency virus. J. Virol. 76(22), 11440-11446 (2002).

45. Wang T, Yang S, Petrenko VA, Torchilin VP. Cytoplasmic delivery of liposomes into MCF-7 breast cancer cells mediated by cell-specific phage fusion coat protein. Mol. Pharm. 7(4), 1149-1158 (2010).

46. Yang B, Ming X, Cao C et al. High-throughput screening identifies small molecules that enhance the pharmacological effects of oligonucleotides. Nucleic Acids Res. 43(4), 1987-1996 (2015).

-• This study identifies UNC10217938A as an endosomal escape enhancing compound.

47. D’Astolfo DS, Pagliero RJ, Pras A et al. Efficient intracellular delivery of native proteins. Cell 161(3), 674-690 (2015).

48. Osteikoetxea X, Sodar B, Nemeth A et al. Differential detergent sensitivity of extracellular vesicle subpopulations. Org. Biomol. Chem. 13(38), 9775-9782 (2015).

49. Chairoungdua A, Smith DL, Pochard P, Hull M, Caplan MJ. Exosome release of beta-catenin: a novel mechanism that antagonizes Wnt signaling. J. Cell Biol. 190(6), 1079-1091 (2010).

50. Perez-Hernandez D, Gutierrez-Vazquez C, Jorge I et al. The intracellular interactome of tetraspanin-enriched microdomains reveals their function as sorting machineries toward exosomes. J. Biol. Chem. 288(17), 11649-11661 (2013).

51. Laplante M, Sabatini DM. mTOR signaling in growth control and disease. Cell 149(2), 274-293 (2012).

52. Thery C, Amigorena S, Raposo G, Clayton A. Isolation and characterization of exosomes from cell culture supernatants and biological fluids. Curr. Protoc. Cell Biol. 30(1), 3.22.1-3.22.29 (2006).

53. Boing AN, Van Der Pol E, Grootemaat AE, Coumans FA, Sturk A, Nieuwland R. Single-step isolation of extracellular vesicles by size-exclusion chromatography. J. Extracell. Vesicles 3, doi:10.3402/jev.v3.23430 (2014).

54. Heath N, Grant L, De Oliveira TM et al. Rapid isolation and enrichment of extracellular vesicle preparations using anion exchange chromatography. Sci. Rep. 8(1), 5730 (2018).

55. Peitz M, Pfannkuche K, Rajewsky K, Edenhofer F. Ability of the hydrophobic FGF and basic TAT peptides to promote cellular uptake of recombinant Cre recombinase: a tool for efficient genetic engineering of mammalian genomes. Proc. Natl Acad. Sci. USA 99(7), 4489-4494 (2002).

56. Madani F, Lindberg S, Langel U, Futaki S, Graslund A. Mechanisms of cellular uptake of cell-penetrating peptides. J. Biophys. 2011, 414729 (2011).

57. Libersou S, Albertini AA, Ouldali M et al. Distinct structural rearrangements of the VSV glycoprotein drive membrane fusion. J. Cell Biol. 191(1), 199-210 (2010).

58. Albertini AA, Merigoux C, Libersou S et al. Characterization of monomeric intermediates during VSV glycoprotein structural transition. PLoS Pathog. 8(2), e1002556 (2012).

59. Mangeot PE, Dollet S, Girard M et al. Protein transfer into human cells by VSV-G-induced nanovesicles. Mol. Ther. 19(9), 1656-1666 (2011)

60. Rohovie MJ, Nagasawa M, Swartz JR. Virus-like particles: next-generation nanoparticles for targeted therapeutic delivery. Bioeng. Transl. Med. 2(1), 43-57 (2017).

61. Lainscek D, Lebar T, Jerala R. Transcription activator-like effector-mediated regulation of gene expression based on the inducible packaging and delivery via designed extracellular vesicles. Biochem. Biophys. Res. Commun. 484(1), 15-20 (2017). 